\title{
Assessing the business impact of Artificial Intelligence
}

\author{
Christian Dietzmann \\ University of Leipzig \\ Business Engineering Institute St. Gallen AG \\ christian.dietzmann@bei-sg.ch
}

\author{
Rainer Alt \\ University of Leipzig \\ rainer.alt@uni-leipzig.de
}

\begin{abstract}
This research introduces two artefacts that contribute to the common understanding of Artificial Intelligence (AI) and aim to provide guidance for designing AI applications. On the one hand, the periodic table of AI structures the broad spectrum of AI technologies and an AI application design model supports the business-oriented conception of AI technologies. Both artefacts are key for the development of an AI impact analysis model to evaluate further organizational impacts and potentials for re-design. The research was motivated by the findings of a survey on AI application examples in a research consortium consisting of German, Swiss and Austrian bank and IT provider managers and a business user group of a Swiss private bank. Both artefacts showed to be helpful tools for change management and IT/business architects.
\end{abstract}

\section{Introduction}

The impact of Artificial Intelligence (AI) is compared with the effects the steam engine had onto the economy and society in the $17^{\text {th }}$ century [1], [2]. It is accepted that $\mathrm{AI}$ and its applications profoundly impact organizations in various ways [3]: By implementing the technology into processes and tasks, AI is reshaping jobs, employment and working environments [4]. To understand the effects of AI on organizations, the assumption is that it not primarily aims to replace tasks or even jobs, but that it rather pursues a human-machine cooperation approach [3], [5]. This task-oriented approach requires well documented reference processes and sub-processes (tasks) as well as an understanding of AI which is based on the human perception of intelligence: The logic behind the latter is that $\mathrm{AI}$ is based on the human experiences with - mostly human intelligence, because AI is developed by humans. Thus, the evaluation of the impacts of $\mathrm{AI}$ on organisations calls for an understanding of both human and $\mathrm{AI}$ intelligence capabilities.

Based on this, the present research develops a competence set of both humans and AI applications or systems and evaluates organizational impacts. As elaborated in chapter 2, it is motivated by a lack of views on $\mathrm{AI}$ from the human intelligence viewpoint and by the potentials of such a perspective on the AI impact analysis. This paper represents the first part of an AI research agenda developed within a research consortium as described in chapter 3. The present study is based on the following overall research question (RQ 1): What is the understanding of AI by digital experts and digital non-experts on the scope of AI? The RQ is specified by three sub RQs. (RQ 1.1): Are both groups able to distinguish between cases in which AI is applied and not applied? (RQ 1.2): Are both groups able to assess the complexity of AI cases? (RQ 1.3): Does previous knowledge or experience on AI influence the decisions of both groups?

Investigating the beforementioned RQs aims to detect and dissolve general misunderstandings and eventual differences in the understanding of $\mathrm{AI}$ between digital experts and non-experts. Two artefacts are proposed and evaluated in chapter 5 to support the alignment of both groups e.g. in AI development and change management projects. Chapter 6 draws a conclusion and formulates further research steps.

\section{Motivation and Background}

The term "Artificial Intelligence" is based on "intelligence", which is known as an abstract term, because even psychologists fail to agree on its definition [6]. The psychological research on human intelligence is determined by various intelligence structure models and approaches towards the explanation of (human) intelligence. Francis Galton lay the fundament of the first intelligence test by Alfred Binet and the following debate on intelligence structure models by defining intelligence as a general cognitive ability on which solving puzzles depends 
[7]. Binet, in contrast, did not assume a general cognitive ability and interpreted intelligence as a set of different abilities [8]. Spearman developed the first intelligence structure model by combining the before mentioned, seemingly opposite approaches to intelligence. He assumed that $g$, the general factor of intelligence, influences the cognitive abilities of a human and is complemented by several factors, such as the processing speed, which impact the human performance in fulfilling specific tasks [9].

From that point on, more sophisticated models like the two-component theory of intelligence by Bernard Cattel, Thurstone's multiple-factors theory and later the theory of multiple intelligences by Howard Gardner have developed which provide a diverse understanding of human intelligence [10], [11], [12]. Cattell divided the $g$ into the fluid intelligence $g_{f}$ and the crystallized intelligence $g_{c}$ and defined them as factors of intelligence. While $g_{f}$ is determined as the ability to adapt to new problems and situations without the need for substantial levels of prior learning, e.g. identify patterns and solve problems, $g_{c}$ represents cognitive skills in which the cumulative effects of previous learning solidified [10]. Thurstone found that $g$ is not central for human intelligence, but several primary mental abilities (PMAs) including verbal comprehension, word fluency, number facility, spatial visualization, associative memory, perceptual speed and reasoning [11]. The theory of multiple intelligences denies the approach of a general cognitive ability and distinguishes between eight abilities of intelligence (musical-rhythmic, visual-spatial, verbal-linguistic, logical-mathematical, bodily-kinesthetic, interpersonal, intrapersonal, naturalistic) and formulated eight criteria to be fulfilled by an intelligence ability [12], [13], [14].

Many AI definitions fall short of considering the before mentioned factors of human intelligence as AI researchers are primarily focused on subfields like machine learning and natural language processing and the underlying technical abilities [15]. To understand $\mathrm{AI}$ and its impacts on interactions in society and the economy, psychological insights on human intelligence need to be included into the approach to AI as proposed by the AI pioneer John McCarthy [16]. Marvin Minsky's idea of AI was not far from the before mentioned considerations, he described AI as "the science of making machines do things that would require intelligence if done by men [and women]" [17]. Especially machine learning and neural networks are increasingly confused with the term AI, although they only technically enable IT systems to learn [18], [19].
Nevertheless, both are currently important subfields of AI which are closely linked to human intelligence, e.g. with the two-component theory of Cattell: While machine learning represents fluid intelligence $g_{f}$, neural networks represent the crystallized intelligence $g_{c}$. Uniting both the psychological and the technical perspective on AI accounts for (a) the possibility of AI technology developing beyond human intelligence in the sense of general AI instead of being a copy of human behavior [20] and (b) the understanding of intelligence from both the human and the machine perspective [21]. In addition, connecting AI technologies with the eight intelligences of Gardner could further deepen the understanding of $\mathrm{AI}$ and the potential impacts of the technology. Defining AI only from the technical viewpoint and based on current fields of application denies the wide spreading possibilities, challenges and nowadays unimaginable functionalities as well as the entire development potential of AI.

\section{Research methodology and design}

The methodology of this paper follows a designoriented approach which is motivated by a digital expert and a digital non-expert survey to identify the research gap. The implications of the survey results are transferred into an artefact which is developed in the context of design-oriented information systems (IS) research [22]. The design-oriented IS research approach suggests a four-step research process containing analysis, design, evaluation and diffusion similar to the design science research guidelines [23].

The research steps are embedded in a consortium research program which started in 2018 and operates until 2022 [24]. The research consortium currently consists of 14 partner companies from Switzerland, Germany and Austria representing the financial value chain, e.g. retail banks, private banks and IT providers. The research consortium representatives mainly comprise digital transformation managers, IT and business architects, project and product managers. Within the research consortium, artefacts are developed in order to foster the application of new technologies in the context of business ecosystems as well as a clear customer and service orientation. Artefacts such as architectures, methods, reference models and tools are co-developed with experts of the consortium. This includes also the definition of research questions in a steering committee and the conception, evaluation and diffusion of possible artefacts and solutions. 


\section{Survey on AI application examples}

The survey contains eight AI examples and was designed to analyze the knowledge level and identify possible misunderstanding patterns on AI within the consortium and between digital experts and nonexperts to derive implications on helpful artefacts for AI development and change management projects. The survey was carried out with 34 members of the research consortium as digital experts and 58 middleand high-level bank employees of a Swiss private bank as digital non-experts. The second group represents potential business users of AI applications designed by the first group.

\subsection{Survey structure}

The participants were presented eight application examples which somehow solved tasks independently or with human input consisting of three games, three business applications, one daily life example and one robotic example. The use cases were shown via video or verbal explanation in connection with pictures or GIFs. The survey participants were provided with an anonymous live-voting tool to ensure unbiased answer on two questions: (1) Is the given example a case of AI or not? (2) How would you estimate the degree of intelligence of the given example? For the second question, a Likert scale with the following five gradations was applied: No intelligence as well as low, medium, high and very high degree of intelligence. After the demonstration of each example, the participants were asked to vote on both questions. An initial definition of AI was not provided since the applied understanding of AI in a real-life setting represented the test object.

\subsection{Example selection, description and answer expectation}

Examples were selected based on (RQ 1.1), (RQ 1.2) and (RQ 1.3) and hence have the intention to test whether (i) participants are able to distinguish between applications which include AI and applications which simply follow rule-based systems or similar, (ii) participants are able to assess the complexity or simplicity of the respective examples and (iii) previous knowledge or experiences influences their decisions.

Example (1) is called Super Mario NEAT and presented as a video with additional verbal explanations. The example name was chosen to clarify that not the game Super Mario itself was meant, but a system that plays the game in a nearly perfect manner. The intention of this example was to test whether the participants recognize that mastering the randomly generated levels requires a definite degree of intelligence. The participants were expected to (a) detect that mastering a randomly generated game environment demands a specific level of intelligence and (b) to hence assign at least a medium degree of intelligence.

Example (2) is called Chess computer of 1996 Deep Blue I and was presented via video. Deep Blue I won two chess games against the former world champion Garry Kasparov in 1996. Although the chess computer finally lost the match against Kasparov 4:2, the successor, Deep Blue II, won the entire match against Kasparov 3.5:2.5 in 1997 [25]. Hence, the IBM developed Deep Blue was the first chess computer that defeated a chess master in a match. Although Deep Blue is enabled by computational power - so-called brute force search instead of knowledge representation and learning, the chess computer is considered a form of AI [15], [25], [26]. It is expected that the participants (a) understand that the computer must evaluate every further move depending on the move of its competitor and (b) that they assign a higher degree of intelligence to the chess computer than to Super Mario NEAT.

Example (3) is AlphaGo, a Google developed AI which is capable of playing the Chinese strategic game Go on master level and to train itself to this level, too [27]. The example is presented with several play move pictures of the match AlphaGo vs. Go master Lee Sedol. Go is considered to be much more complex than chess and that brute force is therefore not applicable [27]. The intention of this example is to test whether the participants understand that Go is much more complex than chess because the Go board is $19 \times 19$ fields compared to the $8 \times 8$ fields chessboard and that hence a higher level of intelligence is required. Furthermore, the complexity of Go is higher because a black or white stone can be put anywhere on the Go board. In such a complex game, brute force is no longer applicable [28]. Hence, the participants are expected to (a) categorize AlphaGo as AI and (b) assign AlphaGo a higher degree of intelligence than examples (1) and (2).

Example (4) is a simple account balancing tool which is shown to the voters as a GIF, simulating that the account holder buys and sells goods while the account balance is continuously calculated every time a transaction is made. It is expected that participants (a) detect that example (4) is no AI and (b) that they assign no degree of intelligence to the application.

Example (5) is a fraud detection application which is shown as a video. The clip shows how the 
application learns to detect fraudulent transactions from test datasets. It is described how data was labelled to make the application detect potentially fraudulent transaction activities. Afterwards, the audience sees how a neural network is structured after the analysis and how it learns about the meaning of the different areas of the neural network in the concrete case. The participants are expected to (a) know that labelling data sets in order to train an algorithm is part of AI development and (b) that such an application represents at least a medium degree of intelligence.

Example (6) is a ticket machine video, which shows the simple step-by-step process of physically buying a train ticket by a human. It is expected that participants (a) understand that the example is a representative of a simple machine and hence is not an example of AI and (b) assign no degree of intelligence.

Example (7) is a video of the humanoid robot "Sophia" developed by Hanson Robotics [29]. The video clip consists of a conversation between Sophia and one of her developers. The audience sees how the robot can instantly react to her conversation partner's questions - even by making jokes and asking senseful counter questions and that it has facial expressions. The survey participants are expected to (a) detect that Sophia's language skills and the spontaneous (counter) reactions require $\mathrm{AI}$ and (b) that the language skills combined with the underlying knowledge represents at least a medium degree of intelligence but not inevitably a high degree of intelligence, because it is only one situation on which she is specifically trained for [30].

Example (8) represents a FAQ chatbot, which is shown as a GIF to the audience and supplemented by verbal explanations on the example. The chatbot is designed for very narrow questions and only responds correctly by clicking on pre-defined questions. Users may also type a question, but if it is not exactly one of the pre-defined questions, the bot issues an error message. Hence, it is expected that participants (a) understand that the FAQ chatbot simply maps answers to pre-defined questions which is not intelligent and (b) that they assign no degree of intelligence, because the bot answers FAQs in such a narrow manner. The expected answers for survey question (1) as well as the applied methods are shown in Table 1.

(RQ 1.1) and (RQ 1.2) apply to all AI application examples, (RQ 1.3) especially applies to examples (1), (2), (4), (6), (7) and (8) as the voting results of these examples might be influenced by prior personal or professional experiences. Example (5) would also be interesting to answer (RQ 1.3), but since only two participants ever worked with a fraud detection tool, prior experiences are not assumed for the voters. Prior experiences are also not assumed for (3), because none of the participants ever played Go. All RQs and especially (RQ 1.3) are analysed by comparing the results of the consortium members' and the Swiss private bank employees' results.

Table 1. Expected answers to survey question (1) and associated methods

\begin{tabular}{|c|c|c|}
\hline Example & Solution & Applied method(s) \\
\hline $\begin{array}{l}\text { (1) } \\
\text { Super } \\
\text { Mario } \\
\text { NEAT }\end{array}$ & $\mathrm{AI}$ & $\begin{array}{l}\text { A* algorithms, rule-based systems and } \\
\text { learning-based controllers using } \\
\text { expression trees, genetic algorithms, } \\
\text { imitation learning, path finding, } \\
\text { reinforcement learning and neural } \\
\text { networks [31] }\end{array}$ \\
\hline $\begin{array}{l}(2) \\
\text { Deep } \\
\text { Blue I }\end{array}$ & AI & $\begin{array}{l}\text { Alpha-beta search (carried out by } 30 \\
\text { IBM RS/ } 6000 S P \text { processors that } \\
\text { searched } 50-100 \text { million chess } \\
\text { positions per second) [15] }\end{array}$ \\
\hline $\begin{array}{l}\text { (3) } \\
\text { AlphaGo }\end{array}$ & AI & $\begin{array}{l}\text { Monte Carlo tree search, reinforcement } \\
\text { learning and deep neural network [28] }\end{array}$ \\
\hline $\begin{array}{l}\text { (4) } \\
\text { account } \\
\text { balancing }\end{array}$ & not AI & Rule-based system \\
\hline $\begin{array}{l}\text { (5) } \\
\text { fraud } \\
\text { detection }\end{array}$ & AI & $\begin{array}{l}\text { Extract of possible methods: Deep } \\
\text { neural network (DNN); decision trees; } \\
\text { particle swarm optimization (PSO), } \\
\text { teaching-learning-based optimization } \\
\text { (TLBO); linear regression, artificial } \\
\text { neural networks (ANN) [33] }\end{array}$ \\
\hline $\begin{array}{l}(6) \\
\text { ticket } \\
\text { machine }\end{array}$ & not AI & Deterministic automation [34] \\
\hline $\begin{array}{l}\text { (7) } \\
\text { Sophia }\end{array}$ & AI & $\begin{array}{l}\text { Face recognition expert system, } \\
\text { supervised and unsupervised learning, } \\
\text { (deep) neural networks, natural language } \\
\text { processing [29] }\end{array}$ \\
\hline $\begin{array}{l}\text { (8) FAQ } \\
\text { chatbot }\end{array}$ & not AI & Binary, rule-based expert system [36] \\
\hline
\end{tabular}

\subsection{Survey results}

The overall results of the survey on the AI application examples confirmed the assumption that the digital expert group gives more correct answers than the non-expert group. Besides the expected results, the survey draws some interesting insights on the (mis)understandings of AI for both digital experts and non-experts. One of the most obvious observations on (RQ 1.1) and (RQ 1.2) is that both groups are not linking games to AI, except the expert group for example (3). Interestingly, both groups do not assign AI to examples (1) and (2). The expert survey results of the research consortium and the non-expert results of the Swiss private bank business users are shown in Figures 1 and 2, respectively. 


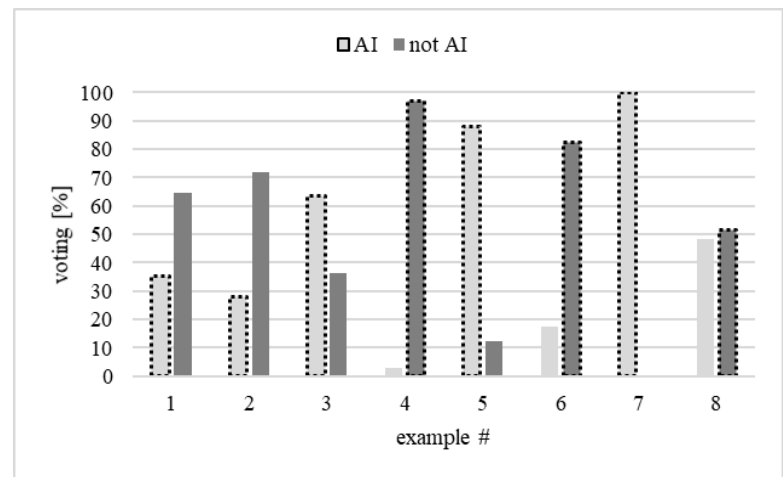

Figure 1. Digital expert group survey results

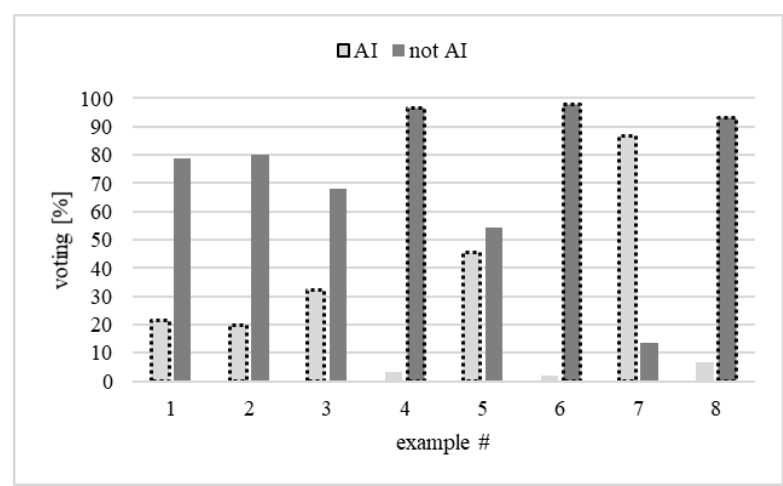

Figure 2. Digital non-expert group survey results

Even though, the estimations on the degree of intelligence reflect that both groups can distinguish between the different game complexities of Super Mario, chess and Go (see Figure 3). The reason for the results of the game examples (1), (2) and (3) may be found in personal experiences: Playing computer games is not considered intelligent as such entertaining games are normally played by children which are seen as less intelligent than adults. This assumption is true with respect to crystallized intelligence which mainly depends on life experience but not in terms of fluid intelligence. These results hence draw a picture of adults being convinced that knowledge is the same as intelligence. Furthermore, both expert and non-expert group are clearly categorizing Sophia as AI and in average assign a high degree of intelligence to the robot. This underlines the assumption that humans assign higher intelligence level to humanoid interfaces in the sense of embodied conversational interfaced [36]. The reason might be that Sophia includes all aspects considered to build an effective human-humanoid interface (HHI), such as perceiving physical aspects like facial expressions with her sensors, reacting correspondingly and being able to communicate verbally and non-verbally with humans as shown in the example video [37]. On the other hand, the example of Sophia shows that humans assign a high level of intelligence to humanoid interfaces, although they cannot take a look into the black box and understand if the natural language skills seen in the video are explicitly trained or if she reacts with the same confidence in other situations.

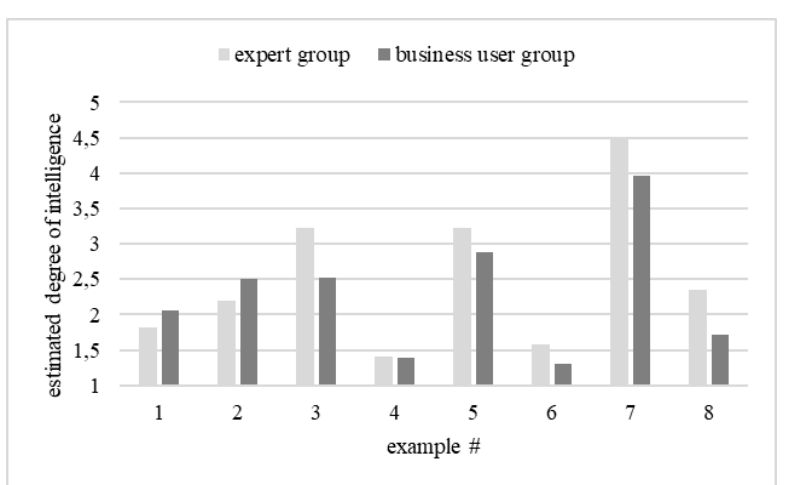

Figure 3. Estimated degree of intelligence

Interestingly, the expert group was divided regarding example (8) FAQ chatbot, while the nonexpert group clearly decided against AI. In addition, the expert group expected a higher degree of intelligence for (8) than the non-expert group. Both groups on average estimated a low degree of intelligence, the estimation of the first group has a tendency towards a medium intelligence degree while the second group had a tendency towards no degree of intelligence. The discussion with the experts revealed that their decision was mainly driven by prior experiences in developing or using a chatbot with a similar interface and that the participants hence automatically assigned a higher degree of intelligence to the chatbot. The hypothesis whereas prior personal or professional experience influences the perception of $\mathrm{AI}$ is underlined by the clear and correct decision of both groups that (4) account balancing and (6) ticket machine are not $\mathrm{AI}$ and that both groups assigned (4) no and (6) nearly no degree of intelligence. Within the non-expert group, in contrast, opinions on example (5) fraud detection differed while experts were sure that this is not an example of AI. Again, it seems that prior experience influences the perception of AI. Even though, both estimated a medium degree of intelligence with the expert group assigning a higher intelligence level.

\subsection{Implications}

The results of the survey imply that knowledge about and better, experience with AI fosters the general understanding of AI. This finding is not surprising, but especially the results on example (1) 
in comparison with (2) and (3) show that adults mostly understand intelligence as crystallized intelligence. Since this assumption is wrong and humans need to enhance their knowledge about intelligence in the forthcoming AI age, an increase of knowledge on intelligence is inevitable. In addition, digital non-experts and (business) users must understand the creation and some basic methods of AI to appropriately use the applications. The second important finding may also be addressed by AI knowledge and more experiences: Humans seemingly tend to assign a higher degree to more human-like interfaces and to interfaces that they have experienced as intelligent before. If more and more people understand possible functionalities behind the black box of AI applications, they will not be misdirected e.g. by interfaces that pretend intelligence. The overall finding of the study is that humans generally need to stay critical and question themselves about the intelligence degree of AI applications. A key aspect to reach such a stadium is to provide both digital experts and digital non-experts or more specifically AI application developers and users with an overview of AI capabilities in order to develop a common understanding on the topic and to build a basis for educational approaches on AI and AI application designing.

\section{Understanding AI functionalities and modeling AI applications}

The periodic table of AI (PTAI) was presented as a possible solution to the research consortium since a literature analysis on the search terms "artificial intelligence structure" and "artificial intelligence understanding" yielded no relevant results containing AI structuring artefacts. The consortium members found the PTAI to dissolve the survey findings and proposed to review and further develop the PTAI by a working group of the consortium consisting of seven digital experts. The group members (a subset of the research consortium) were representatives of two German banks, one German bank IT provider, one Swiss retail bank, one Swiss private bank, one Austrian retail bank and one U.S. technology provider. They are in the middle or top management and oversee technology-driven change management.

\subsection{Periodic table of AI, version 2}

In 2016, Kris Hammond proposed the construct of the PTAI, consisting of 28 elements with each representing one AI functionality and depicting the current variety of AI. His call for advancement of the
PTAI was followed by the German digital association Bitkom e.V. which described every AI element in detail [38].

Interviews on the PTAI with working group members revealed weaknesses like overlaps of several elements and lead to the development of the PTAI, version 2 (PTAI v2) (Figure 4). The elements speech and audio identification were deleted as they were understood as a subset of the elements on recognition. Moreover, Lt (knowledge refinement) was deleted as it is considered a hygiene factor of learning. Ps (Problem solving) was also deleted, because the element was too generic, and the added value remained unclear since every element solves specific problems. Lg (language generation) and $\mathrm{Lu}$ (language understanding) were united to Ln (natural language processing), because the separation was considered too abstract for practical use. The working group also found that the PTAI was lacking information on human intelligence and that the complexity of the elements remained unclear. It was agreed on that supplementing every element with at least one of the eight intelligences defined by Gardner [14] gives an idea what a human would be required to fulfil an AI functionality and generates insights on the complexity. In a first iteration, the AI elements were combined with the corresponding intelligences by the working group members. In the second iteration, the element descriptions were screened and at least one of the eight intelligences was assigned to parts of the sentences. The intelligences were implemented into the PTAI v2 by assigning different colour layers to the elements with each representing one of the intelligences. The more one colour layer is in the front, the more important it is for the specific AI element. The elements were ordered along axis $\mathrm{X}$ based on the AI definition by Stuart Russel and Peter Norvig and supplemented by the dimension of learning. Their agent-based approach consists of the three layers perception, processing and action [19]. Elements were ordered along axis $\mathrm{Y}$ by the number of assigned intelligences: Elements with low complexity are found on the top of with increasing complexity to the bottom. In the sense of the fluid intelligence, the structure of the PTAI v2 allows the implementation of new AI functionalities [10].

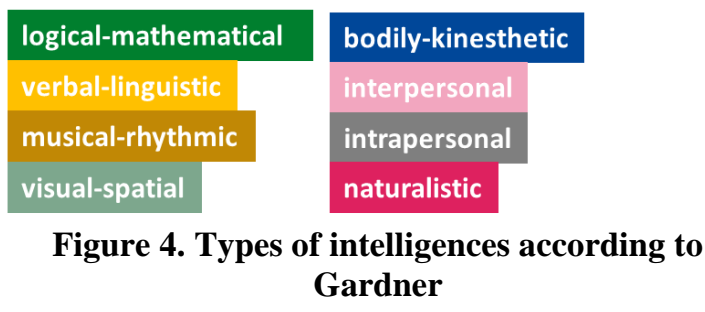




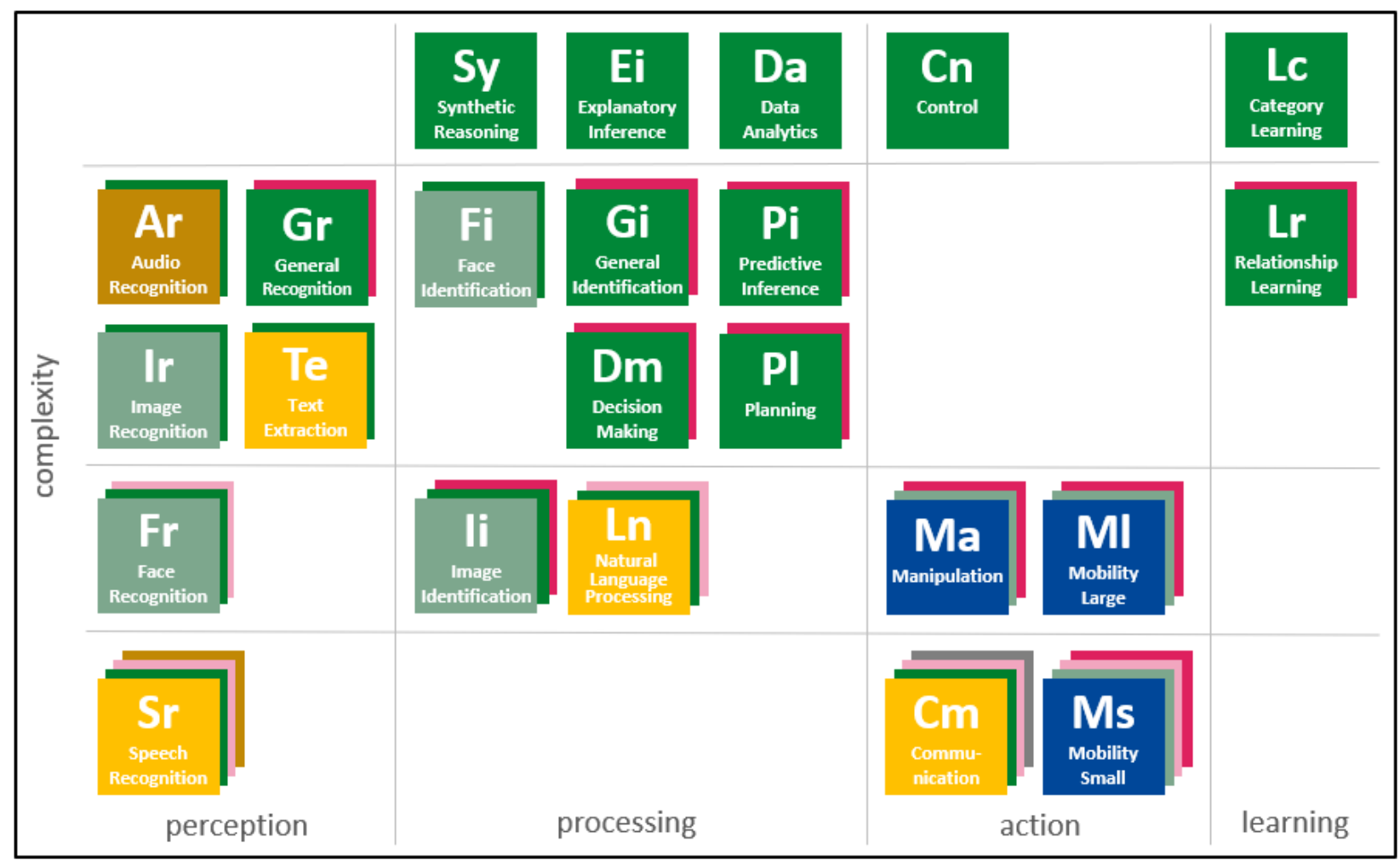

Figure 5. Periodic Table of AI v2 (PTAI v2)

\subsection{AI application design model}

The members of the working group argued that the PTAI v2 educates about AI functionalities and that it is a reasonable starting point for modeling AI applications. For business practice, the construct is transformed in a manner allowing to model concrete use cases. This is done by structuring the AI elements in a 3-step AI application design (AIAD) model which is motivated by the dimensions of the AI definition by Russell and Norvig (Figure 6) [19]. The definition approach by Russel and Norvig is taking the environment into account which is crucial for contextualizing AI applications. Each of the three steps was renamed by the working group due to practical use. Hence, the model is applicable if a task of an existing process or a new task is chosen and if the use case can be described on a functionality level (Figure 7). The elements chosen in the concrete use case of a self-learning chatbot in the task of need finding within a reference bank advisory process have the following definitions [39]:

- Te (text extraction) represents text analysis to extract information about entities, time, places, and facts that are only in the text

- Da (data analytics) represents data analysis to identify specific facts and / or events that represent that data
- Ln (natural language processing) represents the creation of natural language texts and / or explanations based on a certain understanding of the world; Creating a semantic representation of the meaning of a text that shows the context and some understanding of the functioning of the world

- $\mathrm{Cm}$ (communication) represents mechanisms that support the execution of various forms of human-machine communication

- Lc (category learning) represents the detection of new categories of semantic values used on feature collections [38].

The idea of the AIAD model is to abstract the software design process in a manner that includes both AI functionalities and the human counterpart. By modeling an AI use case in the AIAD model, a minimum of one element per step should be chosen which supports high-level requirements definition.

\subsection{Evaluation of the artefacts}

Both the PTAI v2 and the AIAD model were presented to the research consortium on a workshop with 22 participants. Afterwards, the consortium was divided in three groups for the evaluation, which was guided by questions on the evaluation criteria for design science research artefacts [40]. 


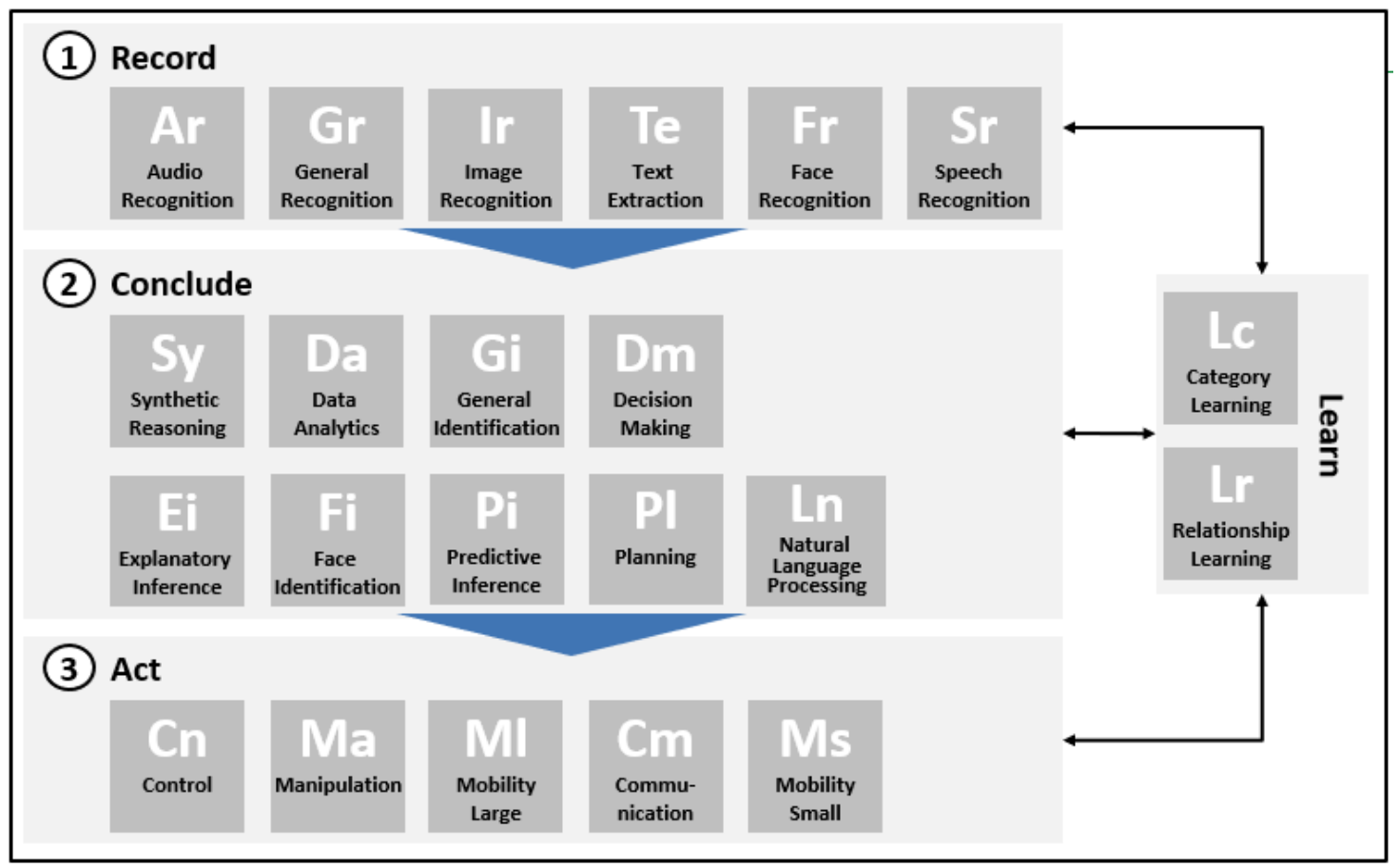

Figure 6. AI application design model (AIAD)

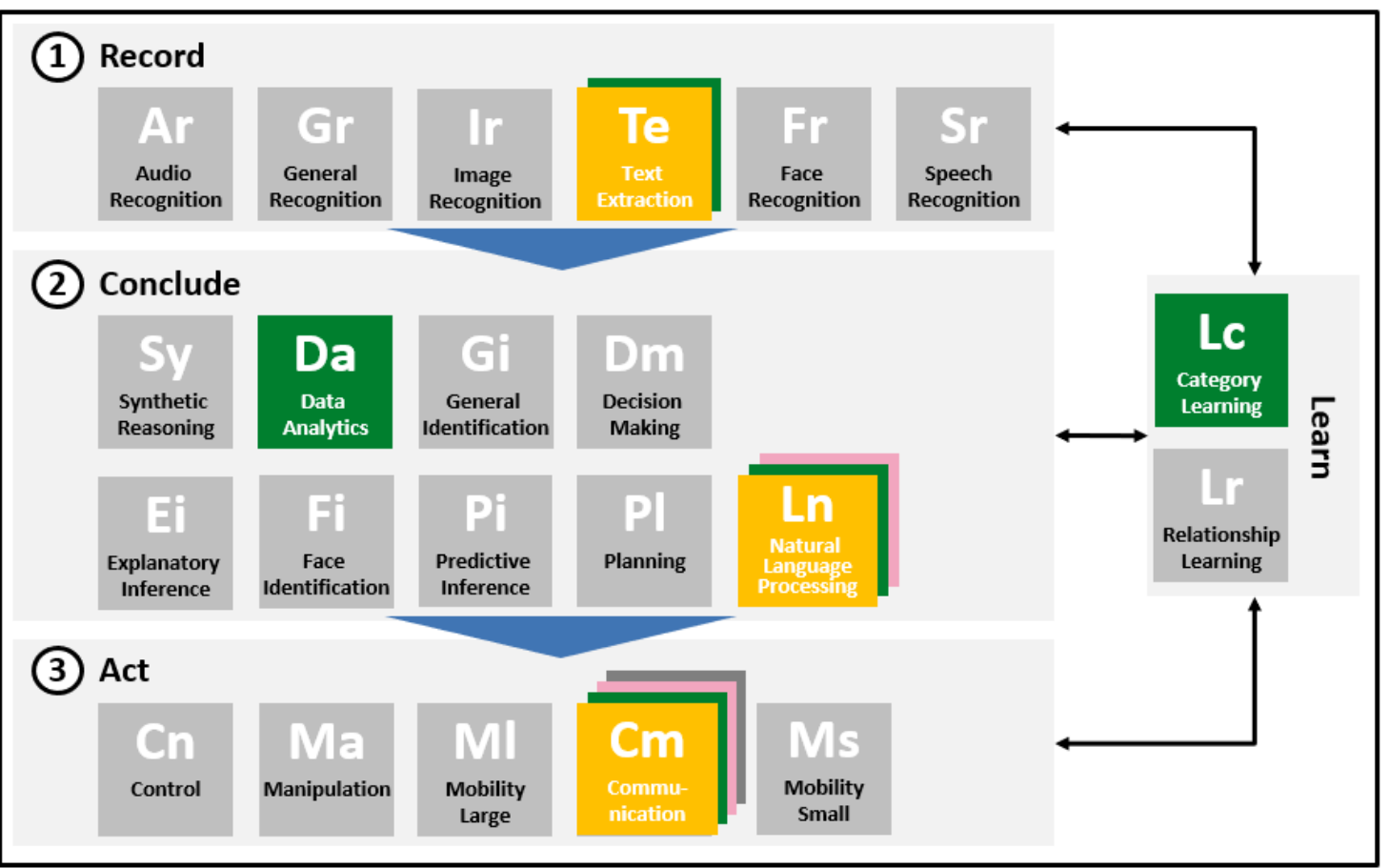

Figure 7. AI application design model (AIAD) applied for a self-learning chatbot in the task of need finding

The participants were asked to rate each criterion from 1 (I completely disagree) to 5 (I totally agree) and write down their thoughts on each. The first task was to give feedback on the PTAI v2 and the second to evaluate the AIAD. For the latter, a use case was provided with the goal of designing a self-learning chatbot in the task of the need finding within a reference bank advisory process [39]. The attribute self-learning is not specified on this level as this would require a deeper technical knowledge on AI methods. Based on the use case, the participants evaluated their experiences with both artefacts. From 
their viewpoint, both perform well, with a slight advantage for the PTAI v2. The latter also performs better in terms of deviation in the mean value. Table 2 and Table 3 show the median of each criterion in grey background.

Table 2. Feedback on PTAI v2

\begin{tabular}{|c|c|c|c|c|c|c|}
\hline \multirow[t]{2}{*}{ Criteria } & \multicolumn{5}{|c|}{ Distribution on the Likert scale [\%] } & \multirow[t]{2}{*}{$\overline{\mathbf{x}}$} \\
\hline & 1 & 2 & 3 & 4 & 5 & \\
\hline $\begin{array}{l}\text { Complete- } \\
\text { ness }\end{array}$ & 0 & 0.091 & 0.364 & 0.455 & 0.091 & 3.5 \\
\hline Ease of use & 0 & 0.227 & 0.364 & 0.364 & 0.045 & 3.2 \\
\hline Elegance & 0 & 0 & 0.5 & 0.41 & 0.091 & 3.6 \\
\hline Simplicity & 0 & 0.045 & 0.273 & 0.545 & 0.136 & 3.8 \\
\hline $\begin{array}{l}\text { Understan- } \\
\text { dability }\end{array}$ & 0 & 0 & 0.182 & 0.454 & 0.364 & 4.2 \\
\hline
\end{tabular}

The results point out that the PTAI v2 supports the understanding on AI and is a simple artefact which is not overengineered. Although evaluation results are satisfactory, emphasis is placed on the detected weaknesses: Obviously, the PTAI v2 fails to meet the participants' demands concerning ease of use and elegance. The normal distribution of the first and the average value of 3.2, are both signals for further improvements on the ease of use. The participants' comments made clear that the PTAI v2 alone is not creating value from a change management or IT/business architecture viewpoint, that the description is required to understand the elements and that a mapping on corresponding software solutions would be helpful. However, it was mentioned that an increased use of the construct will foster the understanding and hence induce value creation.

The evaluation of the AIAD model required other criteria than the PTAI v2 (Table 3 ).

Table 3. Feedback on AIAD model.

\begin{tabular}{llllllll}
\hline Criteria & \multicolumn{6}{c}{ Cistribution on the Likert scale [\%] } & $\overline{\mathbf{x}}$ \\
& $\mathbf{1}$ & $\mathbf{2}$ & $\mathbf{3}$ & $\mathbf{4}$ & $\mathbf{5}$ & \\
\hline $\begin{array}{l}\text { Complete- } \\
\text { ness }\end{array}$ & 0.053 & 0.211 & 0.263 & 0.474 & 0 & 3.2 \\
$\begin{array}{l}\text { Fidelity w. } \\
\text { real world }\end{array}$ & 0 & 0.211 & 0.316 & 0.421 & 0.053 & 3.3 \\
$\begin{array}{l}\text { Consis- } \\
\text { tency }\end{array}$ & 0 & 0.105 & 0.316 & 0.421 & 0.158 & 3.6 \\
$\begin{array}{l}\text { Level of } \\
\text { detail }\end{array}$ & 0 & 0.053 & 0.421 & 0.368 & 0.158 & 3.6 \\
$\begin{array}{l}\text { Robust- } \\
\text { ness }\end{array}$ & 0 & 0.158 & 0.474 & 0.368 & 0 & 3.2 \\
\end{tabular}

As shown in Table 3, participants are not fully convinced by the level of detail and the robustness while they especially value fidelity with the real world and the consistency of the model. The participants argued that the robustness of the model needs to be proved with different use cases and that a higher frequency of using the model will lead to an appropriate level of detail.

\section{Conclusion and outlook}

The results of the present research imply that both digital experts and non-experts connect some misunderstandings with AI, even though experience with AI seems to support the understanding on it. In general, survey participants mostly interpret human intelligence as crystallized intelligence which restricts and influences their view on AI. Hence, they cannot detect that some tasks require fluid intelligence which also misdirects them to believe in seemingly intelligent interfaces. The survey results prove that AI remains a black box without a certain degree of expertise and experience which motivates the introduction of artefacts like the PTAI v2 and the AIAD model. Both proved to be competitive artefacts to increase common understanding of $\mathrm{AI}$ and to design AI applications since both represent a flexible, future-oriented and human-centred approach. This is enabled by a psychological approach in the development of the artefacts: While the PTAI v2 layers perception and action cover functionalities which could be assigned to the crystallized intelligence $g_{c}$, the processing and the learning layer are mostly associated with the fluid intelligence $g_{f}$. Furthermore, the PTAI v2 visualizes the difference between human and AI by applying Gardner's intelligences and already generates one important insight: Most of the complex AI elements cover tasks which can be easily carried out by humans while less complex AI elements pose major difficulties for humans. Therefore, the PTAI v2 seems appropriate to connect the opposite abilities of AI and humans.

The PTAI v2 and the AIAD model are subject to continuous improvement by applying them to bank and other industry use cases to deepen the validity and broaden the spectrum of use. The overall goal of the artefacts is to deepen the understanding of AI, to provide a basis for AI application designing and discuss impacts of AI. The latter will be addressed by an AI impact analysis model based on the PTAI v2 and the AIAD model together with role and task descriptions. The AI impact analysis model aims to assess the impact of specific AI applications on chosen tasks, to derive impact patterns on specific task types as well as needs for organizational change. 


\section{References}

[1] S. Holtel, „Artificial Intelligence Creates a Wicked Problem for the Enterprise“, Procedia Computer Science 99, 2016, pp. 171-180.

[2] McKinsey Global Institute, „Notes from the frontier modelling the impact of AI on the World Economy“, discussion paper, 2018.

[3] S. Makridakis, "The Forthcoming Artificial Intelligence (AI) Revolution: Its Impact on Society and Firms.", Futures 90, 2017, pp. 46-60.

[4] M.-H. Huang and R. T. Rust, „Artificial Intelligence in Service.", Journal of Service Research 21(2), 2018, pp. 155-172.

[5] J.-M. Hoc, „From human - machine interaction to human - machine cooperation“, Ergonomics 43(7), 2000, pp. 833-843.

[6] R.J. Sternberg, Handbook of human intelligence, CUP Archive, 1982.

[7] F. Galton, Hereditary genius: „An inquiry into its laws and consequences“, vol. 27. Macmillan, London, 1869.

[8] A. Binet and V. Henri, „La psychologie individuelle.“, L'année psychologique 2, 1895, pp. 411-465.

[9] C. Spearman, „„,General intelligence“ objectively determined and measured.", The American Journal of Psychology 15(2), 1904, pp. 201-292.

[10] R. B. Cattell, "Abilities: their structure, growth, and action.” Houghton Mifflin, Oxford, 1971.

[11] L. L. Thurstone, „Primary mental abilities“. University of Chicago Press, Chicago, 1938.

[12] H. Gardner, „The theory of multiple intelligence” vol. Annals of Dyslexia 37, 1987, pp. 19-35.

[13] H. Gardner, Multiple intelligences: The theory in practice., Basic Books, New York, 1993.

[14] H. Gardner, Intelligence reframed: Multiple intelligences for the 21st century. Basic Books, New York, 1999.

[15] R. E. Korf, „Does Deep Blue use Artificial Intelligence?", ICGA Journal, 1997, pp. 243-245.

[16] J. McCarthy, „From here to human-level AI“, Artificial Intelligence 171(18), 2007, pp. 1174-1182.

[17] M. L. Minsky, „Semantic Information Processing“, The MIT Press, Cambridge, 1968.

[18] D. Michie, On machine intelligence, Edinburgh University Press, Edinburgh 1968.

[19] S. J. Russell and P. Norvig. Artificial intelligence: a modern approach, Pearson Education, Malaysia, 2016.

[20] B. Goertzel and C. Pennachin, Artificial general intelligence, Springer, Berlin Heidelberg, 2007.

[21] W. L. Johnson, „Needed: A new test of intelligence“, SIGART Bulletin 3(4), 1992, pp.7-9.

[22] H. Österle, J. Becker, U. Frank, T. Hess, D. Karagiannis, H. Krcmar, P. Loos, P. Mertens, A. Overweis, and E. J. Sinz, „Memorandum on design-oriented information systems research", European Journal of Information Systems 1, 2011, pp.7-10.

[23] K. Peffers, T. Tuunanen, M. A. Rothenberger, S. Chatterjee, "A Design Science Research Methodology for Information Systems Research". Journal of Management Information Systems 24(3), 2007, pp. 45-77.
[24] A. Back, G. v. Krogh, E. Enkel, "The CC Model as Organizational Design Striving to Combine Relevance and Rigor", Systemic Practice and Action Research 20(1), 2007, pp. 91-103.

[25] M. Campbell, A. J. Hoane, and F. Hsu, "Deep Blue", Artificial Intelligence 134, 2002, pp. 57-83.

[26] D. DeCoste, "The Future of Chess-Playing Technologies and the Significance of Kasparov Versus deep Blue. In Deep Blue Versus Kasparov", The Significance for Artificial Intelligence, 1997, pp. 9-13.

[27] T. Munakata, "Thoughts on Deep Blue vs. Kasparov", Communications of the ACM. 39(7), 1996, pp. 91-92.

[28] D. Silver, J. Schrittwieser, K. I. Simonyan, Antonoglou, A. Huang, A. Guez, and Chen, Y., "Mastering the game of go without human knowledge", Nature, 550(7676), 2017, p. 354.

[29] J. Retto, "Sophia, first citizen robot of the world", ResearchGate, URL: https://www. researchgate.net.

[30] J. Vincent, "Sophia the robot's co-creator says the bot may not be true AI, but it is a work of art, URL:

https://www.theverge.com/2017/11/10/16617092/ sophia-

the-robot-citizen-ai-hanson-robotics-ben-goertzel,

downloaded on 12.05.2019.

[31] J. Togelius, S. Karakovskiy, and R. Baumgarten, "The 2009 Mario AI Competition", IEEE Congress on Evolutionary Computation, 2010, pp. 1-8.

[32] L. Sterling, and K. Taveter, The Art of Agent-Oriented Modeling, MIT Press, 2009.

[33] D. Choi, and K. Lee, "An Artificial Intelligence Approach to Financial Fraud Detection under IoT Environment: A Survey and Implementation", Security and Communication Networks, 2018, pp. 1-15.

[34] M. O. Rabin, and D. Scott, "Finite automata and their decision problems.", IBM journal of research and development 3(2), 1959, pp. 114-125.

[35] A. Abraham, Rule-Based expert systems. Handbook of measuring system design, 2005.

[36] J. Cassell, T. Bickmore,, M. Billinghurst, L. Campbell, K. Chang, H. Vilhjálmsson, and H. Yan, "Embodiment in conversational interfaces: Rea", Proceedings of the SIGCHI conference on Human Factors in Computing Systems, 1999, pp. 520-527.

[37] K. Kawamura, R. A. Peters, D. M. Wilkes, W. A. Alford, and T. E. Rogers, "ISAC: Foundations in humanhumanoid interaction", IEEE Intelligent Systems and their Applications, 15(4), 2000, pp. 38-45.

[38] Bitkom e.v., Digitalisierung gestalten mit dem Periodensystem der Künstlichen Intelligenz, 2016.

[39] R. Nüesch,, T. Zerndt, R. Alt and R G. Ferretti, "Tablets Penetrate the Customer Advisory Process: A Case from a Swiss Private Bank". BLED 2016 Proceedings, 2016, pp. 45-61.

[40] C. Sonnenberg and J. vom Brocke, "Evaluations in the Science of the Artificial - Reconsidering the BuildEvaluate Pattern in Design Science Research", International Conference on Design Science Research in Information Systems, 2012, pp. 381-397. 